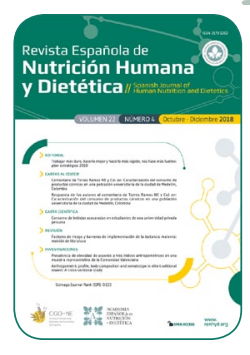

\title{
REVISIÓN
}

\section{Factores de riesgo y barreras de implementación de la lactancia materna: revisión de literatura}

\section{Daiana Catherine Tirano Bernate ${ }^{a, *}$, Olga Lucia Pinzón Espitia ${ }^{a, b, c}$, Javier Leonardo González Rodríguez}

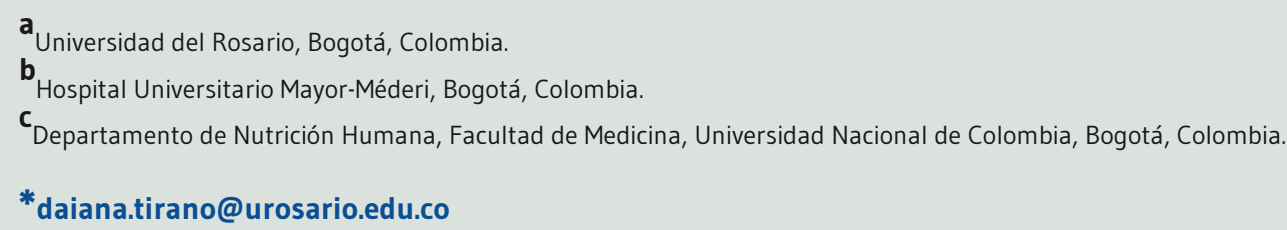

Editor Asignado: Eduard Baladia. Comité Editorial de la Revista Española de Nutrición Humana y Dietética. Pamplona, España

Recibido el 31 de julio de 2017; aceptado el 11 de diciembre de 2018; publicado el 21 de diciembre de 2018.

\section{PALABRAS CLAVE}

Lactancia Materna;

Prevención y control;

Trastornos de la Lactancia.
Factores de riesgo y barreras de implementación de la lactancia materna: revisión de literatura

\section{RESUMEN}

La lactancia materna asegura la adecuada nutrición durante las primeras etapas del desarrollo infantil dadas sus múltiples e insuperables propiedades, por esto es causal de gran impacto para la salud pública mundial; pese a este reconocimiento, se presentan dificultades que obstaculizan su curso normal e incluso producen su abandono. A fin de establecer estrategias que puedan potenciar índices de lactancia materna tales como su duración y exclusividad, es imperativo identificar los retos que los grupos de apoyo a lactancia deben asumir al momento de intervenir en la promoción y manejo de los problemas más frecuentes descritos en la literatura. Tras una revisión exhaustiva de 55 estudios publicados en los últimos cinco años, se identificaron estrategias de prevención y control de los principales problemas asociados con la lactancia materna, además se describió el impacto de ésta en la disminución de la tasa de morbimortalidad mundial a corto mediano y largo plazo, entonces a razón de la protección promoción y apoyo de la lactancia materna se describen 3 políticas públicas principales denominadas: alimentación del lactante y el niño pequeño, hospitales amigos del niño y ley de comercialización de sucedáneos de la leche materna. Es así como los resultados obtenidos ponen en manifiesto la importancia de llevar a cabo intervenciones de promoción mediante transferencia adecuada de información y abordaje de los problemas habituales durante la lactancia a través de grupos de apoyo con el fin de mejorar las prácticas y así prevenir y solucionar posibles problemas potenciales presentes durante esta etapa. 
Risk factors and barriers to implementation of breastfeeding: review

\section{KEYWORDS}

Breast Feeding;

Prevention \& control;

Lactation Disorders.

\section{ABSTRACT}

Breastfeeding ensures adequate nutrition during the early stages of child development, because multiple and unsurpassed properties, which is why it has a great impact on world public health; Despite this recognition, there are difficulties that hinder its normal course and even produce its abandonment. In order to establish strategies that can boost breastfeeding rates such as duration and exclusivity, it is imperative to identify the challenges that breastfeeding support groups must assume, when they are intervening in the promotion and management of the most frequent problems described in the literature. After an exhaustive review of 55 studies published in the last five years, strategies for prevention and control of the main problems associated with breastfeeding were identified, as well as the impact of this on the decrease of the short-term and long term morbidity and mortality rate. In the promotion, proteccion and and support of breastfeeding, 3 main public policies are described: infant and young child feeding, child-friendly hospitals and the marketing law of breast milk substitutes. This is how the results show the importance of carrying out advocacy interventions through adequate transfer of information and addressing the usual problems during breastfeeding through support groups in order to improve practices and thus prevent and solve possible potential problems present during this stage.

\section{CITA}

Tirano Bernate DC, Pinzón Espitia OL, González Rodríguez JL. Factores de riesgo y barreras de implementación de la lactancia materna: revisión de literatura. Rev Esp Nutr Hum Diet. 2018; 22(4): 263-71. doi: 10.14306/ renhyd.22.4.442

\section{INTRODUCCIón}

La lactancia materna (LM) desempeña un papel esencial e irremplazable para una adecuada nutrición durante las primeras etapas de la población infantil. Una vez finalizado el periodo de gestación en condiciones normales, el binomio madre e hijo ha desarrollado mecanismos fisiológicos que permiten dar inicio a la etapa de la lactancia; sus múltiples y bien reconocidos beneficios la catalogan como un objetivo de salud pública a nivel mundial dada su superioridad incuestionable ${ }^{1-3}$.

Existe evidencia de los innumerables beneficios de la $\mathrm{LM}^{3}$, es por esto que la organización mundial de la salud (OMS) y el fondo de las Naciones Unidas para la infancia (UNICEF) apoyan y aconsejan alimentar al recién nacido con leche materna exclusiva durante los primeros 6 meses de vida, y posteriormente de forma complementaria junto a una nutrición adecuada y segura hasta los 2 años o más ${ }^{4-7}$.
La leche materna es el alimento fundamental para el desarrollo y crecimiento del ser humano debido a sus innumerables propiedades nutricionales y funcionales, que no han podido ser replicadas en su totalidad de forma artificial ${ }^{8}$. La leche humana contiene una alta carga nutricional representada por macronutrientes carbohidratos principalmente lactosa, grasa, proteínas y calorías, y micronutrientes como vitaminas, sodio, potasio y yodo.

Al mismo tiempo se tiene la certeza de la presencia de moléculas bioactivas dentro de su contenido las cuales intervienen en diversos procesos fisiológicos e inmunológicos; con factores de reparación y regeneración tisular, antiinflamatorios, antiinfecciosos; con atributos que intervienen en el desarrollo y crecimiento de órganos ${ }^{9}$, en la maduración del sistema inmunológico y colonización de microbiota; además contiene células madre ${ }^{10,11}$, probióticos, enzimas y hormonas ${ }^{12,13}$, inclusive hay datos de propiedades de control del dolor para los recién nacidos por posible presencia de opiáceos endógenos en su contenido ${ }^{14,15}$. 
La leche materna también posee propiedades dinámicas, ya que cambia para satisfacer tanto los requerimientos de nutrición como también los de crecimiento y desarrollo veloz de los lactantes. Complementariamente se han descrito variaciones en la composición de la leche materna que dependen principalmente de los días de posparto, edad gestacional, vaciado del seno, dado que los cambios difieren de una mujer a otra ${ }^{12}$.

Asimismo, se ha demostrado que a través de la inmunización de las madres la concentración de inmunoglobulinas aumenta, entonces el nivel de factores protectores de la LM se intensifica y ha tenido acción directa en las tasas de gripa disminuyéndose ésta en la diada mamá-bebé.

A pesar de su alta importancia, la lactancia materna no ha llegado a niveles óptimos de prevalencia ${ }^{16}$, se tienen datos en los cuales se menciona que sólo un tercio de las madres del mundo llevan a cabo la práctica de la lactancia materna exclusiva (LME) durante los primeros seis meses, esto encierra el $38-40 \%$ de los niños de 6 meses o menos de 6), y tal proporción no ha aumentado durante la última década ${ }^{17,18}$.

Haciendo una delimitación en el territorio mundial, se obtienen los siguientes datos de LME: las mayores tasas se presentan en el sur de Asia con un $60 \%$, en el este y el sur de África (57\%), luego disminuye considerablemente en el oeste del mismo continente (29\%), para Europa se encuentran cifras por debajo del $20 \%$, y para Latinoamérica y el Caribe un $32 \%{ }^{19}$.

Con respecto al panorama en Colombia, cifras mas específicas reportan que para el año 2013 el promedio de LME fue de 1,8 meses y el promedio de LM total de 14,9 meses, las cuales se encuentran muy por debajo al ser comparadas con las directrices internacionales de 6 meses para la LME y de 24 meses o más para la LM total respectivamente ${ }^{20-23}$.

A nivel mundial se establecen medidas estratégicas de promoción, protección y apoyo a la LM con el fin de aumentar su prevalencia, y es importante mencionar que en el marco de acción de las metas mundiales de nutrición para el 2025 instauradas en la asamblea mundial de salud en 2012 se fija como objetivo que la tasa de LM exclusiva en los primeros 6 meses de vida sea incrementada hasta al menos el $50 \%$.

Teniendo en cuenta el abordaje conceptual anterior, y partiendo de la experiencia en la práctica clínica, en la cual es sentida la necesidad de contar con información para los grupos de apoyo, relacionada con consejería en diferentes tópicos relacionados con las dificultades frecuentes que presentan las madres durante su etapa de LM, el propósito de la presente investigación fue identificar los retos para los grupos de apoyo en lactancia materna, sobre la prevención y el manejo de los problemas más frecuentes identificados en la literatura, con el fin de establecer estrategias de intervención que generen un impacto favorable en la práctica de lactancia.

Es de suma importancia ampliar la búsqueda de estrategias para fomentar la educación en lactancia materna, dada la falta de consideración de medidas prioritarias, que fortalezcan el trabajo multidisciplinario en pro de intervenciones que fomenten y protejan la lactancia materna ${ }^{24-26}$.

En la práctica actual, varios autores reportan algunos factores de riesgo y barreras para la una óptima lactancia materna, como son los determinantes multifactoriales, directivas legales y normativas, actitudes y valores sociales, condiciones laborales y servicios de atención médica para permitir que las mujeres amamanten ${ }^{27}$, así como factores analizados desde perspectivas biculturales ${ }^{28}$, y la gestión de rutinas de cuidado durante la cesárea, a fin de brindar apoyo específico a la lactancia materna y promover una mayor duración de la lactancia ${ }^{29}$.

El presente artículo pretende constituirse en un documento orientativo para la prevención y control de los problemas frecuentes durante la lactancia materna a partir de la evidencia documental publicada en los últimos cinco años sobre el tema, pretendiendo dar respuesta a la necesidad de la comprensión de factores de riesgo y barreras para la implementación de una adecuada lactancia materna; considerados como claves en la estrategias de educación de los grupos de apoyo, las cuales se asocian a la disminución de las cifras de prevalencia de LM expuestas anteriormente.

\section{MATERIAL Y MÉTODOS}

Para la realización del presente trabajo se siguieron los principios propuestos por la declaración PRISMA ${ }^{30}$. Se incluyeron publicaciones que circunscribieran el abordaje de las problemáticas asociadas a la lactancia materna dirigidos a mujeres lactantes y publicados en artículos científicos. La presente revisión se llevó a cabo en dos fases:

- La fase 1 incluyó el proceso de selección de los estudios mediante la lectura del título y resumen para identificar la pertinencia del tema y la elegibilidad de los artículos incluidos en la presente revisión, incluido en el protocolo de búsqueda, que permitiera identificar los retos para los grupos de apoyo en lactancia materna, sobre la prevención y el manejo de los factores de riesgo o las barreras de implementación de la lactancia materna más frecuentemente identificados. 
- La fase 2 incluyó la revisión de los textos completos de los artículos preseleccionados y, posteriormente, se aplicaron los criterios de inclusión o exclusión. La calidad metodológica de los artículos seleccionados se evaluó utilizando la Lista de verificación de la metodología de la red escocesa intercolegial (SIGN) http://www.sign. ac.uk, los estudios que se consideraron deficientes se excluyeron para garantizar la calidad de la revisión y minimizar el riesgo de confusión y sesgo.

A partir de los hallazgos encontrados en la revisión, se establecieron las estrategias de prevención y control para ser tenidas en cuenta por los grupos de apoyo que realizan acompañamiento a las madres que presentan dificultades con la lactancia materna.

Los artículos de la presente revisión se identificaron a través de la búsqueda automatizada en la base de datos PubMed y ScienceDirect. Complementariamente, se realizó una búsqueda de literatura disponible en internet en las páginas y recursos electrónicos de organismos intergubernamentales como la OMS, UNICEF y la Organización de las Naciones Unidas para la Alimentación y la Agricultura (FAO), así como en el Sistema Integrado de Búsqueda del Centro de Recursos de Apoyo al Aprendizaje y la Investigación de la Universidad del Rosario (CRAl). La revisión se efectuó entre los meses de enero de 2012 y junio de 2017.

Los descriptores o palabras clave de búsqueda que se utilizaron fueron los siguientes: Lactancia Materna, Lactancia, Prevención \& Control y Desordenes de la Lactancia. Estos términos también se utilizaron en inglés: Breast Feeding, Prevention and Control, Lactation Disorders.

Para la búsqueda de bibliografía se utilizó la siguiente ecuación: ("Breast Feeding"[MeSH Terms] OR ("Breast"[All Fields] AND "Feeding"[All Fields]) OR "Breast Feeding"[All Fields]) AND ("Prevention and Control"); aplicando los siguientes filtros: publicaciones realizadas en los últimos 5 años, idioma español-inglés.

El resultado del proceso de búsqueda realizada por tres autores a partir de la ecuación de búsqueda mencionada permitió seleccionar 55 estudios y documentos que cumplieron con los criterios de selección, así como incluir publicaciones que tuvieran las palabras claves en título y resumen y que en el texto permitiera identificar estrategias de prevención y control de los problemas asociados a la lactancia materna.

En los casos en que los autores consideraron una cuarta opinión para definir la inclusión de algún documento, se consultó a un profesional en nutrición y dietética experto en lactancia materna.

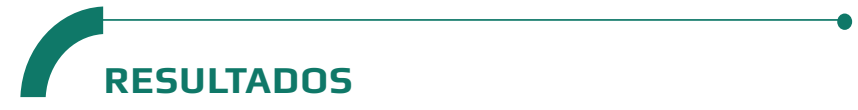

Como resultados, se identificaron un total de 1.086 artículos, de los cuales sólo se incluyeron 430 posteriormente a la revisión de artículos duplicados; en la primera fase de revisión por título y resumen se eliminaron 319 artículos (212 por lectura del título y 107 por lectura del resumen) por no permitir dar respuesta al tema de interés. Posterior a la lectura del texto completo, de los 111 artículos restantes se eliminaron 56 artículos por no cumplir con los criterios de calidad, siendo 55 los artículos incluidos en la revisión.

\section{Lactancia materna como factor protector de enfermedades en la madre y el niño}

La OMS menciona beneficios invaluables de la lactancia materna, entre ellos explica que las madres que lactan exclusivamente reciben un método de anticoncepción que ofrece un $98 \%$ de protección durante los primeros seis meses posparto. Se agrega a esto que facilita el restablecimiento del metabolismo permitiendo la recuperación de su peso8; obtienen beneficios en su sistema cardiovascular ${ }^{8}$; además de reduccir el riesgo de enfermedades tales como cáncer de mama y de ovario, osteoporosis, diabetes de tipo 2 y depresión postparto ${ }^{31}$.

Al mismo tiempo se destaca que la leche materna es el mejor alimento para los lactantes, es biodisponible y adecuadamente aceptada y tolerada por los recién nacidos ${ }^{3}$; contiene el aporte nutricional necesario ${ }^{6}$ y su propiedad de inocuidad junto al alto contenido de anticuerpos atribuye a los infantes una menor posibilidad de adquirir enfermedades como neumonía y diarrea, catalogadas como las dos causas principales de mortalidad en la población mundial infantil ${ }^{6,24}$; además a largo plazo, en los niños que recibieron leche materna, se evidencia menor tendencia de sobrepeso u obesidad ${ }^{3,31,32}$, desnutrición y enfermedades derivadas de ésta ${ }^{33}$, diabetes tipo 2 y relación directa con mejor desarrollo cognitivo $0^{2,31,34-39}$.

Existen datos científicos donde se demuestra que los bebés alimentados con leche materna tienen tasas más bajas de enfermedades gastrointestinales, respiratorias y alergias; adicionalmente mejor agudeza visual y menor riesgo de muerte súbita del lactante ${ }^{40}$.

Paralelamente, por la presencia de efectos potenciales derivados de la exposición a la contaminación del ambiente, se atribuye también que la leche materna contiene factores de protección para dicha contaminación ${ }^{41}$. 
En conclusión, la LM permite gozar del nivel más alto de salud posible tal y como se reconoce en la Convención sobre los Derechos del Niño, y si se aumentara a índices exitosos, se salvarían las vidas de más de 820.000 niños menores de 5 años y 20.000 mujeres que, actualmente conforman la tasa anual de mortalidad global ${ }^{42,43}$.

\section{Directrices para la prevención y el manejo de problemas asociados a la lactancia materna}

En el marco de acción de las metas mundiales de nutrición para el 2025, que se establecieron en la asamblea mundial de salud en 2012, se fija como objetivo que la tasa de LM exclusiva en los primeros 6 meses de vida sea incrementada hasta al menos el 50\% para el año $2025^{6}$, esto conlleva un gran esfuerzo a nivel colectivo donde se involucra a los gobiernos, las sociedades, y los sistemas de salud.

Actualmente los responsables de la formulación de medidas legislativas en todos los países del mundo centran su atención en mantener, mejorar y exigir el cumplimiento de normas y políticas que potencien la LM, además en la creación y perfeccionamiento de nuevas estrategias para el logro del objetivo mundial y, adicionalmente, comprender y hacer extensivo el contexto de la importancia a gran escala de la lactancia.

Existen múltiples estrategias de apoyo a la LM, entre ellas: la Estrategia Mundial para la Alimentación del Lactante y del
Niño Pequeño ${ }^{6}$, la iniciativa de hospitales amigos del niño y la reglamentación de comercialización de sucedáneos de la leche materna; con las cuales también se ofrece protección a su curso natural ${ }^{44}$.

Proteger y fomentar la LM es un punto crítico sujeto a mejorar en materia económica; el impacto en la salud de las prácticas de lactancia materna según recomienda la OMS y UNICEF sumaría alrededor de 300.000 millones de dólares al año a la economía mundial. De este modo, el aumento de las tasas de lactancia materna reduciría significativamente los costos a las familias y a los gobiernos en el tratamiento de enfermedades infantiles ${ }^{45}$. Además, y apuntando al desarrollo y evolución de los seres humanos, éstos contarían con mejores características que les permitirían un desempeño óptimo en la sociedad.

Es así como la lactancia materna es una de las medidas de salud más costo-efectivas ${ }^{17}$; siendo por ello susceptible de intervención, con miras a resultados eficaces, que evite siempre la reducción en la frecuencia y duración de ésta ${ }^{46,47}$.

Las estrategias de prevención y control de problemas presentados durante la lactancia materna mitigan el efecto indeseado de su abandono o el suministro temprano de fórmulas lácteas que la sustituyen, los cuales son expuestos a continuación en la Tabla 1.

Tabla 1. Estrategias de prevención y control de problemas frecuentes de la lactancia materna.

\section{PROBLEMA}

Información deficiente o errada a las madres y familias sobre los beneficios irremplazables de la lactancia y el impacto positivo a corto, mediano y largo plazo de ésta ${ }^{6}$.

Apoyo y acompañamiento suficiente que permita resolver dudas e interrogantes acerca del proceso, además anticiparse a posibles problemas $3,6,8,49,50$.

\section{ESTRATEGIAS DE PREVENCIÓN Y CONTROL}

- Garantizar a las madres información específica, oportuna, en lenguaje claro y preciso acerca de la lactancia materna, sus beneficios y su impacto favorecedor en la salud en los diferentes lapsos de tiempo: corto, mediano y largo plazo ${ }^{13,48}$.

- Realizar promoción de la lactancia materna preferiblemente por consejeros calificados en salud y brindar acompañamiento disponible en cualquier situación que se pueda presentar.

- Proveer a los padres de herramientas facilitadoras para la anticipación y búsqueda de soluciones a los problemas relacionados con la lactancia. 


\section{PROBLEMA}

Abordaje de principales dificultades en el binomio madre-hijo, como posición inadecuada del bebé, llanto o irritabilidad y hambre percibida, percepción de baja producción láctea, dolor en los senos, congestión mamaria, grietas del pezón y/o mastitis ${ }^{1,3,8,16,31,40,50-52}$.

\section{ESTRATEGIAS DE PREVENCIÓN Y CONTROL}

- Desarrollar intervenciones educativas con metodología verbal y práctica que permitan la identificación y abordaje de las principales dificultades relacionadas con la lactancia en el binomio madre-hijo.

- Prevenir las dificultades frecuentes que se presentan durante los primeros días de lactancia materna y se atribuyen a manifestaciones físicas y fisiológicas del binomio madre-hijo.

- Asegurar el aprendizaje de la madre en la técnica adecuada para lactar; esto incluye: posición correcta y cómoda, prestando atención especial a problemas de los senos y pezones, además colocación adecuada del complejo pezónareola en la boca del bebé.

- Garantizar apoyo profesional e idóneo para abordar los desfíos y ayudar a las madres a cumplir con la duración òptima de la lactancia materna.

Incapacidad o falta de autoeficacia para resolver problemas asociados a la lactancia 27,53 .

- Empoderar a las madres en el proceso de lactancia materna, logrando así que obtengan mejor percepción de sus capacidades organizativas y de acción de para lactar.
- Preparar a las madres para la reincorporación laboral, brindando herramientas y estrategias a ser usadas en sus sitios de trabajo que permitan la continuidad de la lactancia materna y proteger el derecho a amamantar.

Las madres que no amamantan con éxito son menos propensas a intentar la lactancia materna en los embarazos posteriores ${ }^{55}$. en lactancia de las madres, convirtiendo de esta forma a las posteriores prácticas en satisfactorias y amigables.
- Realizar actividades de apoyo desde la primera experiencia

Fuente: Elaboración propia de los autores.

\section{DIscusión}

Se han expuesto anteriormente múltiples razones que justifican la importancia de la lactancia materna; entonces, no alimentar a los bebés con leche materna exclusiva, o realizar prácticas inapropiadas de lactancia en sus primeros 6 meses de vida, supone 1,4 millones de muertes y el $10 \%$ del total de enfermedades que se presentan en menores de 5 $\operatorname{años}^{18,56}$

Prestar atención especial a la promoción, protección y prevalencia de la LM contribuye al control de la morbimortalidad del mundo permitiendo un alto impacto en la salud y por ende en la mejora de las condiciones y calidad de vida de los seres humanos. Debido a lo mencionado anteriormente se señala la necesidad de adaptar las estrategias de apoyo a la lactancia materna, ya que al llevar a cabo intervenciones optimas de promoción $\mathrm{n}^{47,57}$ se puede potenciar significativamente la lactancia aumentando su duración y exclusividad, además permitiendo resolver problemas asociados a ésta ${ }^{58}$.

En relación con los grupos de apoyo para la intervención de problemas asociados a la lactancia materna, es muy frecuente que las madres experimenten dificultades durante la lactancia, y la falta de conocimiento o tener establecidos conceptos errados puede generar sentimientos de frustración, que al no ser mitigados y/o asesorados de la forma correcta pueden causar un efecto desfavorecedor para la LM, conllevando a la reducción en su frecuencia y su duración ${ }^{59}$.

Las múltiples fuentes de información para los padres, relacionada con LM, como publicidad, internet, familiares y amigos, personal de salud y otros, pueden facilitar el pobre fundamento sobre la salud o confusión conceptual. Esto puede cambiar las percepciones y debilita la confianza depositada, causando por ejemplo el retiro de la lactancia. 
Múltiples estrategias educativas que consisten en proporcionar toda la información posible y pertinente a los padres y sus familias acerca de la LM por parte de profesionales de la salud con experiencia certificada han demostrado la eficacia en la prevalencia de la $L M^{31}$. Las diversas intervenciones como el apoyo a través de soporte telefónico ${ }^{49}$, terapias de grupo o consejerías a domicilio se sostienen con un buen nivel de evidencia; el cual demuestra que, cuando se lleva a cabo la asesoría en el hogar y en la comunidad, resultan en un aumento del $85 \%$ en las tasas de inicio precoz de lactancia y por ende debe recibir la más alta prioridad.

Es muy importante unificar conceptos entre los responsables del cuidado de las mujeres en etapa de gestación y posparto, ya que dentro de las instituciones de salud deben participar en la correcta educación al binomio madre-hijo y su familia para, de esta forma, hacerlos poseedores de información realista y no idealista ${ }^{27}$ que facilite a las madres cumplir con la duración deseada de la lactancia materna.

Un factor destacado para mejorar la percepción de apoyo, y que permite el empoderamiento de las madres y sus familias, es el fomento de habilidades suficientes para prevenir y contrarrestar los problemas frecuentes de la LM, los cuales se pueden presentar en los primeros 10 días posparto, lapso en el cual es ideal la intervención integra 127,60.

El presente artículo demuestra la necesidad por parte de las madres y sus familias de recibir la mayor cantidad de educación posible en materia de lactancia materna a través de diferentes estrategias, y aunque se analizaron los factores relevantes, se esperaba evidenciar información que manifestara algunos resultados de las intervenciones educativas ya puestas en marcha, lo cual no ocurrió debido a la obtención de estudios y comunicación selectiva encontrada, esto dado a los criterios de información seleccionados por los autores.

En base a lo anterior se sugiere incentivar el seguimiento y/o medición de las intervenciones ejecutadas, también realizar búsqueda exhaustiva de información que permita identificar el impacto de la educación en lactancia a través del tiempo con cifras y estadísticas.

\section{CONCLUSIONES}

La lactancia materna tal cual es sugerida por la OMS cumple con su efecto protector de enfermedades, lo cual impacta directamente a la tasa de morbimortalidad a corto, mediano y largo plazo en los individuos y, por consiguiente, para la sociedad, constituyéndose en una medida costo-efectiva que impulsa la economía mundial. Las prácticas de lactancia materna responden de manera efectiva a las intervenciones de grupos de apoyo, en la medida que permiten aumentar las cifras de prevalencia de la lactancia materna exclusiva garantizando su continuidad. La presente revisión pone en evidencia cómo las intervenciones llevadas a cabo para mejorar las prácticas de lactancia permiten responder a problemas sensibles y que se pueden mejorar representativamente. Las estrategias propuestas deben ser llevadas a cabo por todos los actores posibles, y deben ser impulsadas y replicadas desde los directivos políticos, legales y económicos de los países; asimismo, la sociedad, involucrando a las familias para crear un vínculo sólido que ofrezca toda la protección y respaldo posible en pro de la lactancia. Se sugiere realizar estudios de indagación de experiencias vividas por parte de las madres y núcleos familiares que recibieron intervención en lactancia materna, con el fin de evaluar el impacto real de las intervenciones realizadas.

\section{$\longrightarrow$ AGRADECIMIENTOS \\ Los autores expresan su agradecimiento a la Universidad del Rosario. \\ $\longrightarrow$ CONFLICTO DE INTERESES}

Los autores expresan que no existen conflictos de interés al redactar el manuscrito.

\section{REFERENCIAS}

(1) Tedder J. The Roadmap to Breastfeeding Success: Teaching Child Development to Extend Breastfeeding Duration. J Perinat Educ. 2015; 24(4): 239-48.

(2) Victora CG, Horta BL, Loret de Mola C, Quevedo L, Pinheiro RT, Gigante DP, et al. Association between breastfeeding and intelligence, educational attainment, and income at 30 years of age: a prospective birth cohort study from Brazil. Lancet Glob Health. 2015; 3(4): e199-205.

(3) Aguilar Cordero M], Sánchez López AM, Madrid Baños N, Mur Villar N, Expósito Ruiz M, Hermoso Rodríguez E. Lactancia materna como prevención del sobrepeso y la obesidad en el niño y el adolescente; revisión sistemática. Nutr Hosp. 2015; 31(2): 606-20.

(4) Calvo Quirós C. Factores socioeconómicos, culturales y asociados al sistema de salud que influyen en el amamantamiento. Enferm Actual Costa Rica. 2008; (15): 4. 
(5) Asociación Española de Pediatría. Manual de Lactancia Materna. Madrid: Ed. Médica Panamericana; 2015. 504 p.

(6) Organización Mundial de la Salud. Metas mundiales de nutrición 2025: documento normativo sobre lactancia materna. Organizasión Mundial de la Salud; 2017. Report No.: WHO/NMH/NHD/14.7. Disponible en: http://apps.who.int/ iris/handle/10665/255731

(7) Brown JE. Nutrition Through the Life Cycle. 6 ed. Boston, MA: Cengage Learning; 2016.

(8) Aguilar Cordero M], Madrid Baños N, Baena García L, Mur Villar N, Guisado Barrilao R, Sánchez López AM. Lactancia materna como método para prevenir alteraciones cardiovasculares en la madre y el niño. Nutr Hosp. 2015; 31(5): 1936-46.

(9) Serrao F, Papacci P, Costa S, Giannantonio C, Cota F, Vento $G$, et al. Effect of Early Expressed Human Milk on Insulin-Like Growth Factor 1 and Short-Term Outcomes in Preterm Infants. Plos One. 2016; 11(12): e0168139.

(10) Amitay EL, Keinan-Boker L. Breastfeeding and Childhood Leukemia Incidence: A Meta-analysis and Systematic Review. JAMA Pediatr. 2015; 169(6): e151025.

(11) Mosca F, Giannì ML. Human milk: composition and health benefits. Pediatr Med Chir. 2017; 39(2): 155.

(12) Ballard O, Morrow AL. Human milk composition: nutrients and bioactive factors. Pediatr Clin North Am. 2013; 60(1): 49-74.

(13) Boer S, Unal S, van Wouwe JP, van Dommelen P. Evidence Based Weighing Policy during the First Week to Prevent Neonatal Hypernatremic Dehydration while Breastfeeding. Plos One. 2016; 11(12): e0167313.

(14) Harrison D, Reszel J, Bueno M, Sampson M, Shah VS, Taddio $A$, et al. Breastfeeding for procedural pain in infants beyond the neonatal period. Cochrane Database Syst Rev. 2016; 10: CD011248.

(15) Paixão MJG. Breastfeeding for Procedural Pain in Infants Beyond the Neonatal Period. Clin Nurse Spec. 2018; 32(3): 116-7.

(16) Suresh S, Sharma KK, Saksena M, Thukral A, Agarwal R, Vatsa $M$. Predictors of breastfeeding problems in the first postnatal week and its effect on exclusive breastfeeding rate at six months: experience in a tertiary care centre in Northern India. Indian J Public Health. 2014; 58(4): 270-3.

(17) Díaz-Gómez NM, Ruzafa-Martínez M, Ares S, Espiga I, De Alba C, Díaz-Gómez NM, et al. Motivaciones y barreras percibidas por las mujeres españolas en relación a la lactancia materna. Rev Esp Salud Publica. 2016; 90: e1-18.

(18) Sankar M], Sinha B, Chowdhury R, Bhandari N, Taneja S, Martines ], et al. Optimal breastfeeding practices and infant and child mortality: a systematic review and meta-analysis. Acta Paediatr. 2015; 104(467): 3-13.

(19) Comité de Lactancia Materna de la Asociación Española de Pediatría. Lactancia materna en cifras: tasas de inicio y duración de la lactancia en España y en otros países. Madrid: Asociación Española de Pediatría; 2016. Disponible en: https://www. aeped.es/sites/default/files/documentos/201602-lactanciamaterna-cifras.pdf

(20) Ortiz YMB, Navarro CC, Ruíz GG. Lactancia materna exclusiva: ¿la conocen las madres realmente? Rev Cuidarte. 2014; 5(2): 723-30.

(21) González-Ruiz GE, Reyes-Rios LA, Borre-Ortiz YM, OviedoCórdoba HR, Ditta LB, Carbonó L, et al. Factores que inciden en el destete temprano en un grupo de madres de Santa Marta, Colombia. Investig Enferm Imagen Desarr. 2015; 17(2): 37-50.

(22) Cabrera GA, Mateus JC, Carvajal R. Conocimientos acerca de la lactancia materna en el Valle del Cauca, Colombia. Rev Fac Nac Salud Pública. 2003; 21(1): 27-41.

(23) Neufeld L, Rubio M, Pinzón L, Tolentino L. Nutrición en Colombia: Estrategia de país 2011-2014. Washington, D.C.: Banco Interamericano de Desarrollo; 2010. Disponible en: https://publications.iadb.org/en/publication/13668/ nutricion-en-colombia-estrategia-de-pais-2011-2014

(24) Albarenque S, Mas L, Ferreyra M, Marchisio Ml, Gomila A, Armelini P. Lactancia materna y alimentación complementaria. Arch Argent Pediatr. 2005; 103(3): 257-8.

(25) Zapata ME, Fortino J, Palmucci C, Padros S, Palanca E, Venesia $A$, et al. Diferencias en las prácticas de lactancia materna y alimentación complementaria, según los indicadores básicos propuestos por la OMS, en niños con diferentes condiciones sociodemográficas de Rosario, Argentina. Diaeta. 2015; 33(150): 12-20

(26) Romero-Velarde E, Villalpando-Carrión S, Pérez-Lizaur AB, Iracheta-Gerez M de la L, Alonso-Rivera CG, López-Navarrete $\mathrm{GE}$, et al. Consenso para las prácticas de alimentación complementaria en lactantes sanos. Bol Med Hosp Infant Mex. 2016; 73(5): 338-56

(27) Rollins NC, Bhandari N, Hajeebhoy N, Horton S, Lutter CK, Martines JC, et al. Why invest, and what it will take to improve breastfeeding practices? Lancet. 2016; 387(10017): 491-504.

(28) Stuart-Macadam P, Dettwyler KA. Breastfeeding: biocultural perspectives. New York: Routledge; 2017.

(29) Cato K, Sylvén SM, Lindbäck J, Skalkidou A, Rubertsson C. Risk factors for exclusive breastfeeding lasting less than two months-Identifying women in need of targeted breastfeeding support. Plos One. 2017; 12(6): e0179402.

(30) Urrútia G, Bonfill X. Declaración PRISMA: una propuesta para mejorar la publicación de revisiones sistemáticas y metaanálisis. Med Clin. 2010; 135(11): 507-11.

(31) Chowdhury R, Sinha B, Sankar MJ, Taneja S, Bhandari N, Rollins N, et al. Breastfeeding and maternal health outcomes: a systematic review and meta-analysis. Acta Paediatr. 2015; 104(467): 96-113.

(32) Kwame Sundaram J, Rawal V, Clark MT. Ending malnutrition: from commitment to action. Roma: Food and Agriculture Organization of the United Nations (FAO); 2015. Disponible en: http://www.fao.org/3/a-i4921e.pdf

(33) Branca F, Ellis C. 25 Global and National Public Health Nutrition Approaches. En: Buttriss JL, Welch AA, Kearney JM, LanhamNew SA, editores. Public Health Nutrition. 2 ed. Hoboken, NJ: Wiley-Blackwell; 2017. p. 359-72.

(34) Meedya S, Fahy K, Kable A. Factors that positively influence breastfeeding duration to 6 months: A literature review. Women Birth. 2010; 23(4): 135-45.

(35) Sinha B, Chowdhury R, Sankar MJ, Martines J, Taneja S, Mazumder $S$, et al. Interventions to improve breastfeeding outcomes: a systematic review and meta-analysis. Acta Paediatr. 2015; 104(S467): 114-34

(36) Patnode CD, Henninger ML, Senger CA, Perdue LA, Whitlock EP. Primary Care Interventions to Support Breastfeeding: Updated Evidence Report and Systematic Review for the US Preventive 
Services Task Force. Jama. 2016; 316(16): 1694-705.

(37) Horta BL, Mola CL de, Victora CG. Breastfeeding and intelligence: a systematic review and meta-analysis. Acta Paediatr. 2015; 104(S467): 14-9.

(38) Giugliani ERJ, Horta BL, Mola CL de, Lisboa BO, Victora CG. Effect of breastfeeding promotion interventions on child growth: a systematic review and meta-analysis. Acta Paediatr. 2015; 104(S467): 20-9.

(39) Kim SK, Park S, Oh J, Kim J, Ahn S. Interventions promoting exclusive breastfeeding up to six months after birth: A systematic review and meta-analysis of randomized controlled trials. Int ] Nurs Stud. 2018; 80: 94-105.

(40) Rocca R M, Bosch F J, Henson D C, Reyes H P, Conde A M, Risso $R M$, et al. Adherence to recommendations to reduce the risk of Sudden infant Death Syndrome. Rev Chil Pediatr. 2014; 85(4): 462-9.

(41) Santana Porbén S. Sobre el estado de las prácticas de alimentación del lactante y del niño pequeño descrito según los estándares del Fondo de las Naciones Unidas para la Infancia. Rev Cubana Aliment Nutr. 2017; 27(1 Supl): S125-33.

(42) Martínez-Galán P, Martín-Gallardo E, Macarro-Ruiz D, Martínez-Martín E, Manrique-Tejedor J, Martínez-Galán $P$, et al. Educación prenatal e inicio de la lactancia materna: Revisión de la literatura. Enferm Univ. 2017; 14(1): 54-66.

(43) Ramakrishnan U, Imhoff-Kunsch B, Martorell R. Maternal Nutrition Interventions to Improve Maternal, Newborn, and Child Health Outcomes. En: Black RE, Singhal A, Uauy R, editores. Nestlé Nutrition Institute Workshop Series. Basilea: $S$. KARGER AG; 2014. p. 71-80.

(44) Boskabadi H, Bagheri S. Comparison between infants receiving traditional supplements (camel thorn, flix weed, and sugar water) and exclusively breast fed infants. Avicenna J Phytomed. 2015; 5(6): 479-84.

(45) Gorrita Pérez RR. La lactancia materna, un desafío aún no superado. Rev Cubana Pediatr. 2014; 86(3): 270-2.

(46) Bellù $R$, Condò M. Breastfeeding promotion: evidence and problems. Pediatr Med Chir. 2017; 39(2): 53-6.

(47) Food and Agriculture Organization of the United Nations. Regional Overview of Food Insecurity Asia and the Pacific. Towards a Food Secure Asia and the Pacific. Bangkok: FAO; 2015. Disponible en: http://www.fao.org/3/a-i4624e.pdf

(48) Amir LH, Jones LE, Buck ML. Nipple pain associated with breastfeeding: incorporating current neurophysiology into clinical reasoning. Aust Fam Physician. 2015; 44(3): 127-32.
(49) Fox R, McMullen S, Newburn M. UK women's experiences of breastfeeding and additional breastfeeding support: a qualitative study of Baby Café services. BMC Pregnancy Childbirth. 2015; 15: 147.

(50) Kent JC, Gardner H, Geddes DT. Breastmilk Production in the First 4 Weeks after Birth of Term Infants. Nutrients. 2016; 8(12).

(51) Berger-Larrañaga M, Bustamante-Abuid C, Díaz-Vergara S, Tresierra-Cabrera J, Mayta-Tristán P, Segura ER. Trastornos de la lactancia materna y otros factores asociados a la pérdida de peso neonatal excesiva en un hospital de la Seguridad Social en Lima, Perú. Nutr Hosp. 2015; 32(5): 2062-70.

(52) Odom EC, Li R, Scanlon KS, Perrine CG, Grummer-Strawn L. Reasons for Earlier Than Desired Cessation of Breastfeeding. Pediatrics. 2013; 131(3): e726-32.

(53) Anzola M, Peña-Rosas JP. Metas globales de la Organización Mundial de la Salud para mejorar la nutrición materna, del lactante y del niño pequeño. An Venez Nutr. 2014; 27(1): 26-30.

(54) Sack R. Lactancia materna y trabajo: ¿un derecho reconocido a la mujer? Aljaba. 2015; 19: 117-34.

(55) Lucchini Raies C, Márquez Doren F, Rivera Martínez MS. "Yo quiero amamantar a mi hijo": Develando la experiencia de mujeres que enfrentaron dificultades en su proceso de lactancia. Rev Chil Pediatr. 2017; 88(5): 622-8.

(56) Marco Alegría TD, Martínez Martínez D, Muñoz Gómez M], Sayas Ortiz I, Oliver-Roig A, Richart-Martínez M. Valores de referencia españoles para la versión reducida de la Escala de Autoeficacia para la Lactancia Materna BSES-SF. Anales Sis San Navarra. 2014; 37(2): 203-11.

(57) Victora CG, BahI R, Barros AJD, França GVA, Horton S, Krasevec J, et al. Breastfeeding in the 21st century: epidemiology, mechanisms, and lifelong effect. Lancet. 2016; 387(10017): 475-90.

(58) Patel R, Oken E, Bogdanovich N, Matush L, Sevkovskaya Z, Chalmers B, et al. Cohort profile: The promotion of breastfeeding intervention trial (PROBIT). Int J Epidemiol. 2014; 43(3): 679-90.

(59) Mullen SM, Marshall A, Warren MD. Statewide Breastfeeding Hotline Use Among Tennessee WIC Participants. J Nutr Educ Behav. 2017; 49(7S2): S192-S196.e1.

(60) Heidari Z, Kohan S, Keshvari M. Empowerment in breastfeeding as viewed by women: A qualitative study. J Educ Health Promot. 2017; 6: 33 